TRANSACTIONS OF THE

AMERICAN MATHEMATICAL SOCIETY

Volume 354, Number 1, Pages 107-122

S 0002-9947(01)02863-X

Article electronically published on August 20, 2001

\title{
APS BOUNDARY CONDITIONS, ETA INVARIANTS AND ADIABATIC LIMITS
}

\author{
XIANZHE DAI
}

\begin{abstract}
We prove an adiabatic limit formula for the eta invariant of a manifold with boundary. The eta invariant is defined using the Atiyah-PatodiSinger boundary condition and the underlying manifold is fibered over a manifold with boundary. Our result extends the work of Bismut-Cheeger to manifolds with boundary.
\end{abstract}

\section{INTRODUCTION}

The $\eta$-invariant, introduced by Atiyah-Patodi-Singer in their seminal work APS, is the correction term (from the boundary) for the index formula on a manifold with boundary. The adiabatic limit refers to the geometric degeneration in which the metric is been blown up along certain directions. The study of the adiabatic limit of the $\eta$-invariant is initiated by E. Witten [W], who relates the adiabatic limit of the $\eta$-invariant to the holonomy of determinant line bundle, the so-called "global anomaly". Witten's result was given full mathematical treatment in [BF1, BF2] and [C1], see also [DF, [Si].

In [BC1], J.-M. Bismut and J. Cheeger studied the adiabatic limit for a general fibration of closed manifolds. (The case of the holonomy theorem as studied in $[\mathrm{W}$, [BF1, BF2 and [C1 corresponds to a fibration over the circle.) What they found is that the adiabatic limit of the $\eta$-invariant of a Dirac operator on the total space is expressible in terms of a canonically constructed differential form, $\tilde{\eta}$, on the base. The Bismut-Cheeger $\tilde{\eta}$ form is a higher dimensional analogue of the $\eta$-invariant for it is exactly the boundary correction term in the Families Index Theorem for manifolds with boundary, [BC2, BC3], see also [MP1, MP2] for generalizations. In their work Bismut-Cheeger assumes the invertibility of the Dirac family along the fibers. The more general case is discussed in [D] using the analysis developed in $[\mathrm{MM}$. The adiabatic limit formula is used in [BC4 to prove a generalization of the Hirzebruch conjecture on the signature defect (cf. [ADS], [Mu1]). Other applications of adiabatic limit can be found in $[\mathrm{Z}], \overline{\mathrm{DZ}}]$ and $[\mathrm{N}]$.

In this paper we study the limiting behavior of the $\eta$-invariant of a Dirac operator on the total space of a fibration, when the base space is a compact spin manifold with boundary. We impose the boundary conditions of APS type and restrict ourself to the case when the Dirac family along the fibers is invertible. Our main result is an extension of the Bismut-Cheeger's adiabatic limit formula for the eta invariant.

Received by the editors November 8, 2000.

2000 Mathematics Subject Classification. Primary 58Jxx.

Supported in part by NSF Grant DMS 9704296. 
More precisely, let

$$
Y \rightarrow X \stackrel{\pi}{\rightarrow} B
$$

be a fibration where the fiber $Y$ is closed but the base $B$ may have nonempty boundary. For simplicity we assume that both the fibration and the base are spin, although our discussion extends to the more general case of Clifford modules. Let $g_{B}$ be a metric on $B$ which is of the product type near the boundary $\partial B$. Now equip $X$ with a submersion metric $g$,

$$
g=\pi^{*} g_{B}+g_{Y}
$$

so that $g$ is also product near $\partial X$. This is equivalent to requiring $g_{Y}$ to be independent of the normal variable near $\partial B$, given by the distance to $\partial B$.

The adiabatic metric $g_{x}$ on $X$ is given by

$$
g_{x}=x^{-2} \pi^{*} g_{B}+g_{Y},
$$

where $x$ is a positive parameter.

Associated to these data we have in particular the total Dirac operator $D_{x}^{X}$ on $X$, the boundary Dirac operator $D_{x}^{\partial X}$ on $\partial X$, and the family of Dirac operators $D_{Y}$ along the fibers. If the family $D_{Y}$ is invertible, then, according to [BC1], the boundary Dirac operator $D_{x}^{\partial X}$ is also invertible for all small $x$, therefore the eta invariant of $D_{x}$ with the APS boundary condition, $\eta\left(D_{x}\right)$, is well-defined.

Theorem 1.1. Consider the fibration $Y \rightarrow X \rightarrow B$ as above. Assume that the Dirac family along the fiber, $D_{Y}$, is invertible. Consider the total Dirac operator $D_{x}^{X}$ on $X$ with respect to the adiabatic metric $g_{x}$ and let $\eta\left(D_{x}^{X}\right)$ denote the eta invariant of $D_{x}^{X}$ with the APS boundary condition. Then the limit $\lim _{x \rightarrow 0} \bar{\eta}\left(D_{x}^{X}\right)=$ $\lim _{x \rightarrow 0} \frac{1}{2} \eta\left(D_{x}\right)$ exists in $\mathbb{R}$ and

$$
\lim _{x \rightarrow 0} \bar{\eta}\left(D_{x}\right)=\int_{B} \hat{A}\left(\frac{R^{B}}{2 \pi}\right) \wedge \tilde{\eta},
$$

where $R^{B}$ is the curvature of $g_{B}, \hat{A}$ denotes the $\hat{A}$-polynomial and $\tilde{\eta}$ is the $\eta$-form of Bismut-Cheeger [BC1] (cf. the next section).

This extends the result of Bismut-Cheeger [BC1] to manifolds with boundary. It should be noted that the formula is exactly the same as that of Bismut-Cheeger, which means that the boundary does not contribute in this case.

The main problem here is dealing with the boundary conditions. In fact, there does not seem to be a direct relation between the APS boundary conditions on the base manifold and that on the total space. As a result, one of the main steps, i.e., a uniform bound on the eigenvalues of $D_{x}^{X}$, is much more involved than the one in the case without boundary.

As was mentioned earlier, for closed manifolds, the most general result is obtained in D. Namely, the Dirac family along the fiber is not necessarily invertible, but has kernels of constant dimension. The correponding case for manifolds with boundary is considerably more complicated because of the issue of boundary conditions. In this case the APS boundary condition is no longer self-adjoint and one has to choose a finite rank perturbation. This choice may have to be made to be compatible with the corresponding choice on the base. We intend to return to this issue later.

The paper is organized as follows. We first introduce the $\tilde{\eta}$-form of BismutCheeger. Then we turn to the APS type boundary conditions and establish a number of estimates that are needed in our proof. In $\S 4$ we note an interesting 
property of the APS type boundary conditions, namely, that of the finite propagation speed. Finally we present a uniform estimate of the eigenvalues and the proof of the main result.

Acknowledgement. We would like to thank John Lott for useful conversations. Part of this work was done while the author was visiting IHES and he thanks the Institute for its hospitality.

\section{The $\tilde{\eta}$ Form of Bismut-CheEger}

In this section we recall the situation for closed manifolds, while introducing necessary notations for later sections.

Let

$$
Y \rightarrow M^{n} \stackrel{\pi}{\rightarrow} B^{p}
$$

be a smooth fibration of closed manifolds. A connection determines a splitting of the tangent bundle of $M$ into its vertical subbundle and horizontal subbundle,

$$
T M=T^{V} M \oplus T^{H} M .
$$

Let $P^{H}, P^{V}$ denote the projections on $T^{H}, T^{V}$ respectively relative to this splitting. We will use $U$ 's to denote the vertical vectors on $M$ and $X$ 's the tangent vectors on $B$ as well as their horizontal lifts to $M$.

Now equip $M$ with a submersion metric $g$ which preserves the splitting (2.2),

$$
g=\pi^{*} g_{B}+g_{Y} .
$$

The Levi-Civita connection on $M$ will be denoted by $\nabla^{L}$. This connection does not preserve the splitting (2.2). In [B] a unitary connection $\nabla$ is defined which respects the splitting. In fact,

$$
\nabla_{U_{1}} U_{2}=P^{V}\left(\nabla_{U_{1}}^{L} U_{2}\right), \nabla_{X} U=P^{V}\left(\nabla_{X}^{L} U\right), \nabla_{U} X=0, \nabla_{X_{1}} X_{2}=\nabla_{X_{1}}^{B} X_{2},
$$

where $\nabla^{B}$ denotes the Levi-Civita connection on $B$. Denote by $T$ the torsion tensor of $\nabla$ and $S$ the difference tensor of $\nabla^{L}$ and $\nabla$.

Assume from now on that $\operatorname{dim} M=n$ is odd. Further, assume that both $M$ and $T^{V} M$ are spin. Consequently, so is $B$. The spinor bundle $F(M)$ of the total space is related to $F(B)$, the spinor bundle of the base, and $F(Y)$, the spinor bundle of $T^{V} M$, in the following way:

$$
F(M)=\pi^{*} F(B) \otimes F(Y) .
$$

Let $\xi$ be a hermitian bundle on $M$ with unitary connection $\nabla^{\xi}$ and curvature $L^{\xi}$, and let $\nabla^{u}$ denote the connection on $F(M) \otimes \xi$ defined by

$$
\nabla^{u}=\nabla-\frac{1}{2} \sum\left\langle S\left(e_{i}\right) e_{i}, \cdot\right\rangle
$$

where $\left\{e_{i}\right\}$ is a local orthonormal basis for $T^{V} M$. The Dirac operator along the fiber $D_{Y}$ is defined as $D_{Y}=e_{i} \nabla_{e_{i}}$. Consider the adiabatic metric $g_{x}$

$$
g_{x}=x^{-2} \pi^{*} g_{B}+g_{Y},
$$

where $x$ is a positive parameter. We have the following nice formula for $D_{x}$ [BC1. $(4.26)]$. 
Lemma 2.1. If $\left\{f_{\alpha}\right\}$ is a local orthonormal basis for $T B$, then

$$
D_{x}=x \sum_{\alpha} f_{\alpha} \nabla_{f_{\alpha}}^{u}+D_{Y}-\frac{x^{2}}{4} \sum_{\alpha \leq \beta} f_{\alpha} f_{\beta} T\left(f_{\alpha}, f_{\beta}\right) .
$$

For simplicity, we will denote $\tilde{D}_{B}=\sum_{\alpha} f_{\alpha} \nabla_{f_{\alpha}}^{u}, c(T)=\sum_{\alpha \leq \beta} f_{\alpha} f_{\beta} T\left(f_{\alpha}, f_{\beta}\right)$.

We now recall the definition of the Bismut superconnection $[B]$. The smooth sections of $F(Y) \otimes \xi$ can be viewed as the smooth sections of an infinte dimensional vector bundle $H_{\infty}$ over $B$. The fiber of $H_{\infty}$ over a point $y \in B$ is the space of smooth sections of $\left.(F(Y) \otimes \xi)\right|_{\pi^{-1}(y)}$. Thus it comes with a natural $L^{2}$-metric. The connection $\nabla^{u}$ induces a unitary connection $\tilde{\nabla}^{u}$ on $H_{\infty}$. If we wish to regard the operator $D_{x}$ as acting on sections of $F(B) \otimes H_{\infty}$, we simply write $\tilde{\nabla}^{u}$ for $\nabla^{u}$ in (2.4). The (rescaled) Bismut superconnection $B_{t}$ on $H_{\infty}$ is the superconnection

$$
B_{t}=\tilde{\nabla}^{u}+t^{1 / 2} D_{Y}-\frac{c(T)}{4 t^{1 / 2}}
$$

It follows from (2.4) that $\frac{1}{x} D_{x}$ is the Dirac operator coupled to the Bismut superconnection $B_{x^{-2}}$.

The asymptotics of kernels associated to the Bismut superconnection exhibit some remarkable cancellations. The first one is expressed in the local index theorem for families, [B], [BF2]. Essential to our discussion, however, are the other two cancellation results, [BC1]; i.e. when $\operatorname{dim} Y=2 l$ is even

$$
\operatorname{tr}_{s}\left[\left(D_{Y}+\frac{c(T)}{4 t}\right) e^{-B_{t}^{2}}\right]=O\left(t^{1 / 2}\right) \text { as } t \rightarrow 0,
$$

and when $\operatorname{dim} Y=2 l-1$ is odd

$$
t r^{\text {even }}\left[\left(D_{Y}+\frac{c(T)}{4 t}\right) e^{-B_{t}^{2}}\right]=O\left(t^{1 / 2}\right) \text { as } t \rightarrow 0,
$$

where $t r^{e v e n}$ indicates taking the even form part of $t r$.

Remarkably, when $\operatorname{ker} D_{Y}$ is a vector bundle on $B$, the expressions on the left hand sides of (2.6), (2.7) are also well behaved for the large time. In fact, it is shown in $[\mathrm{BGV}]$ (in a more general setting) that for $\operatorname{dim} Y=2 l$,

$$
\operatorname{tr}_{s}\left[\left(D_{Y}+\frac{c(T)}{4 t}\right) e^{-B_{t}^{2}}\right]=O\left(t^{-1}\right) \text { as } t \rightarrow \infty,
$$

and for $\operatorname{dim} Y=2 l-1$,

$$
\operatorname{tr}^{\text {even }}\left[\left(D_{Y}+\frac{c(T)}{4 t}\right) e^{-B_{t}^{2}}\right]=O\left(t^{-1}\right) \text { as } t \rightarrow \infty .
$$

By virtue of (2.6) (2.9) we now define a differential form on $B$, the $\hat{\eta}$ form

$$
\hat{\eta}= \begin{cases}\frac{1}{\sqrt{\pi}} \int_{0}^{\infty} t r_{s}\left[\left(D_{Y}+\frac{c(T)}{4 t}\right) e^{-B_{t}^{2}}\right] \frac{d t}{2 t^{1 / 2}} & \text { if } \operatorname{dim} Y=2 l, \\ \frac{1}{\sqrt{\pi}} \int_{0}^{\infty} t r^{e v e n}\left[\left(D_{Y}+\frac{c(T)}{4 t}\right) e^{-B_{t}^{2}}\right] \frac{d t}{2 t^{1 / 2}} & \text { if } \operatorname{dim} Y=2 l-1,\end{cases}
$$

assuming that $\operatorname{ker} D_{Y}$ does define a vector bundle on $B$. For example, the first integral is convergent at 0 because of (2.6), and convergent at $\infty$ because of (2.8).

The $\hat{\eta}$ should be viewed as a higher dimensional analogue of the $\eta$-invariant. In fact, when $\operatorname{dim} Y=2 l-1$ is odd, its 0 -form component is exactly the $\eta$-invariant of the Dirac operator along the fiber. Moreover, results of [BC3, BC4] show that it is 
precisely the boundary correction term in the Families Index Theorem for manifolds with boundary. We also point out that when $\operatorname{dim} Y=2 l$ the 1 -form component of $\hat{\eta}$ represents the Bismut-Freed connection of the determinant line bundle $\operatorname{det} D_{Y}^{+}$, which is interpreted by Witten as the covariant anomaly, W]; see also [BF2], [C2], and $\mathrm{DF}$.

We normalize $\hat{\eta}$ by defining

$$
\tilde{\eta}= \begin{cases}\sum \frac{1}{(2 \pi i)^{j}}[\hat{\eta}]_{2 j-1} & \text { if } \operatorname{dim} Y=2 l, \\ \sum \frac{1}{(2 \pi i)^{j}}[\hat{\eta}]_{2 j} & \text { if } \operatorname{dim} Y=2 l-1 .\end{cases}
$$

Here we decompose the odd (respectively even) form $\hat{\eta}$ into its homogeneous components $[\hat{\eta}]_{2 j-1}$ (respectively $[\hat{\eta}]_{2 j}$ ).

Remark. Using the notion of spectral section, whose existence is equivalent to the vanishing of the (odd) analytic index of the family $D_{Y}$, Melrose-Piazza defined a generalization of the Bismut-Cheeger $\tilde{\eta}$ form and proved the corresponding family index theorem, MP1 MP2.

Finally we point out that in the construction of $\tilde{\eta}$ we only need the fiber $Y$ being closed, which is exactly our situation.

\section{ETA INVARIANT OF APS TYPE BOUNDARY CONDITIONS}

There are currently quite a few different approaches to the theory of eta invariant on manifolds with boundary. Cheeger $\mathrm{C} 2$ is the first to define an eta invariant on manifolds with boundary, by coning off the boundary. In [DW] DouglasWoijciechowski defined an eta invariant using the APS boundary condition. This is generalized in $[\mathrm{DF}]$ to deal with the family situation. Melrose $[\mathrm{M}]$ defined an eta invariant in the context of the $b$-calculus. Klimek-Woijciechowski $[\mathrm{KW}$, and independently Müller [Mu2], then defined an eta invariant using manifolds of cylindrical ends, which turns out to be the same as the so-called $b$-eta invariant of Melrose. Furthermore, in [Mu2, Müller proved the equivalence of the APS eta and the cylindrical eta (and hence $b$-eta). It is expected that all of these theories are essentially equivalent. We take the approach of APS boundary conditions.

Consider a compact odd dimensional spin manifold $X$ with boundary, and suppose $X$ has a Riemannian structure which is of product type near the boundary.

Let

$$
D_{X}: C^{\infty}(X, S) \rightarrow C^{\infty}(X, S)
$$

be the formally self-adjoint Dirac operator acting on the spinor bundle $S \rightarrow X$. Then in a collar neighborhood $[0,1) \times Y$ of the boundary, $D$ takes the form

$$
D_{X}=J\left(\partial_{u}+D_{\partial X}\right)
$$

where $J=c(d u)$ and

$$
D_{\partial X}: C^{\infty}\left(Y,\left.S\right|_{Y}\right) \rightarrow C^{\infty}\left(Y,\left.S\right|_{Y}\right)
$$

is the self-adjoint Dirac operator on $Y$ under the identification $\left.S\right|_{Y} \cong S(Y)$.

As an unbounded operator in $H_{X}=L^{2}(X, S)$ with domain $C_{0}^{\infty}(X, S), D_{X}$ is symmetric. (In other words, $D_{X}$ is formally self-adjoint.) To obtain self-adjoint extensions of $D_{X}$, one has to impose boundary conditions. For our purpose, we would like to restrict our attentions to boundary conditions of elliptic type. Appropriate boundary conditions that are of elliptic type are considered by Atiyah-Patodi-Singer 
APS. Namely if we denote by $\Pi_{+}$the orthogonal projection of $L^{2}\left(Y,\left.S\right|_{Y}\right)$ onto the subspace spanned by the eigensections of $D_{\partial X}$ with nonnegative eigenvalues, then $D_{X,+}=D_{X}$ with domain

$$
\operatorname{dom}\left(D_{X,+}\right)=\left\{\varphi \in H^{1}(X, S) \mid \Pi_{+}\left(\left.\varphi\right|_{Y}\right)=0\right\}
$$

is an elliptic boundary value problem (in the generalized sense, see [APS], [Se]). $D_{X,+}$ is now a closed symmetric extension of $D_{X}$, although, in general, $D_{X,+}$ is still not self-adjoint. However, one can obtain elliptic self-adjoint boundary value problems by further considering self-adjoint extensions of $D_{X,+}$.

More generally, fix a nonnegative number $a$ such that $a^{2} \notin \operatorname{spec}\left(D_{\partial X}^{2}\right)$. Consider the orthogonal projection $\Pi_{-a}$ of $H_{\partial X}=L^{2}(\partial X, S)$ onto the direct sum of eigenspaces of $D_{\partial X}$ with eigenvalues greater than $-a$. Consider the operator $D_{a}=D$ with domain given by

$$
\operatorname{dom}\left(D_{a}\right)=\left\{\varphi \in H^{1}(X, S) \mid \Pi_{-a}\left(\left.\varphi\right|_{Y}\right)=0\right\} .
$$

As is proved in $\left[\mathrm{DF}, D_{a}\right.$ is a closed symmetric operator on $H_{X}$ whose self-adjoint extensions turn out to be parametrized by isometries

$$
T: K_{\partial X}^{+}(a) \longrightarrow K_{\partial X}^{-}(a),
$$

where $K_{\partial X}(a)=K_{\partial X}^{+}(a) \oplus K_{\partial X}^{-}(a)$ is the direct sum of eigenspaces of $D_{\partial X}^{2}$ with eigenvalues less than $a$ and the $\mathbb{Z}_{2}$-grading is given $J$. Namely, one has [DF]

Proposition 3.1. There is a one-one correspondence between

$$
\left\{\text { self-adjoint extensions of } D_{a}\right\} \leftrightarrow\left\{\text { unitary maps } T: K_{\partial X}^{+}(a) \rightarrow K_{\partial X}^{-}(a)\right\} \text {. }
$$

For a unitary map $T$, its corresponding self-adjoint extension $D(a, T)$ is given by $D$ with

$$
\operatorname{dom}(D(a, T))=\left\{\varphi \in H^{1}(X, S) \mid\left(\Pi_{a}+\Pi_{T}\right)\left(\left.\varphi\right|_{Y}\right)=0\right\},
$$

where $\Pi_{T}$ is the orthogonal projection onto the graph of $T$ in $K_{\partial X}(a)$.

Thus for any choice $\langle a, T\rangle$ of boundary conditions the Dirac operator $D_{X}(a, T)$ is self-adjoint and elliptic.

Most of the properties we need for the operator $D(a, T)$ can be obtained by studying its heat kernel. And, to construct the heat kernel of $D(a, T)$, we use the gluing construction of APS. For this purpose, we first consider the model situation on the infinite half cylinder $\mathbb{R}_{+} \times \partial X$. In this case, $D=J\left(\partial_{u}+D_{\partial X}\right)$ and we have a global decomposition.

$$
\begin{aligned}
L^{2}\left(\mathbb{R}_{+} \times \partial X, S\right) & =L^{2}\left(\mathbb{R}_{+}, L^{2}\left(\partial X,\left.S\right|_{\partial X}\right)\right) \\
& =L^{2}\left(\mathbb{R}_{+}, K_{\partial X}(a)\right) \oplus L^{2}\left(\mathbb{R}_{+},\left(K_{\partial X}(a)\right)^{\perp}\right) .
\end{aligned}
$$

Since both $D$ and the boundary condition are diagonal with respect to this decomposition,

$$
e^{-t D^{2}(a, T)}=E_{<a}(t)+E_{>a}(t)
$$

splits into two pieces as well. As the boundary condition on $L^{2}\left(\mathbb{R}_{+},\left(K_{\partial X}(a)\right)^{\perp}\right)$ is given by $\Pi_{a}$ which is completely analogous to the APS boundary condition, $E_{>a}(t)$ can be given an explicit formula, as follows. 
Let $\left\{\varphi_{\lambda} ; \lambda \in \operatorname{spec} D_{\partial X}, \lambda>a\right\}$ be an orthonormal basis for $\operatorname{Im}_{a}$ consisting of eigensections of $D_{\partial X}$. Then the same construction in [APS gives

$$
\begin{aligned}
E_{>a}(t)=\sum_{\lambda>a}\left\{\frac{e^{-\lambda^{2} t}}{\sqrt{4 \pi t}}\left(e^{-(u-v)^{2} / 4 t}-e^{-(u+v)^{2} / 4 t}\right) \varphi_{\lambda} \otimes \varphi_{\lambda}^{*}\right. \\
+\left[\frac{e^{-\lambda^{2} t}}{\sqrt{4 \pi t}}\left(e^{-(u-v)^{2} / 4 t}+e^{-(u+v)^{2} / 4 t}\right)\right. \\
\left.\left.\quad-\lambda e^{\lambda(u+v)} \operatorname{erfc}\left(\frac{u+v}{2 \sqrt{t}}+\lambda \sqrt{t}\right)\right] J \varphi_{\lambda} \otimes\left(J \varphi_{\lambda}\right)^{*}\right\},
\end{aligned}
$$

where erfc is the complimentary error function

$$
\operatorname{erfc}(x)=\frac{2}{\sqrt{\pi}} \int_{x}^{\infty} e^{-\xi^{2}} d \xi
$$

On the other hand, while there is no explicit formula for $E_{<a}(t)$, it is reduced to a heat kernel on the half line $\mathbb{R}_{+}$, with the $L^{2}$ boundary condition at $\infty$ and a local boundary condition at 0 :

$$
\left\{\begin{aligned}
\left(\partial_{t}-\partial_{u}^{2}+A^{2}\right) E_{<a}(t, u, v) & =0, \\
\left.E_{<a}\right|_{t=0} & =\mathrm{Id}, \\
\left.\Pi_{T} E_{<a}\right|_{u=0} & =0, \\
\left.J \Pi_{T} J\left(\partial_{u}+A\right) E_{<a}\right|_{u=0} & =0,
\end{aligned}\right.
$$

where $A=\left.D_{\partial X}\right|_{K_{\partial X}(a)}$ is a finite dimensional symmetric endomorphism. Thus its properties are well developed in the standard theory.

From this explicit description, we derive the following important estimate concerning the heat kernel on the infinite half cylinder.

Lemma 3.2. The heat kernel $e^{-t D^{2}(a, T)}$ is smooth in all variables for $t>0$. Moreover, there exists a constant $C$ such that

$$
\left|e^{-t D(a, T)^{2}}\right| \leq C\left(t^{-\frac{n}{2}}+t^{-\frac{1}{2}}\right) \mid e^{-\frac{(u-v)^{2}}{4 t}} .
$$

Proof. The estimate for $E_{>a}(t)$ follows from exactly the same argument of APS, Proposition 2.21], while that for $E_{<a}(t)$ is standard. Now one applies the same argument to $\left(\partial_{t}^{2}+D^{2}(a, T)\right) e^{-t D^{2}(a, T)}$ and iterate.

We also note the following result, which will be used in a crucial way later in our discussion (cf. [DW, Lemma 3.1]).

Lemma 3.3. Assume that $\operatorname{ker} D_{\partial X}=0$ and denote $D=D(0,0)$. For any $u \in \mathbb{R}_{+}$,

$$
\int_{\{u\} \times \partial X} \operatorname{tr}\left(J e^{-t D^{2}}\right)=0, \quad \int_{\{u\} \times \partial X} \operatorname{tr}\left(D e^{-t D^{2}}\right)=0 .
$$

Proof. For $D=D(0,0), e^{-t D^{2}}$ is given by the explicit formula (3.3). Now since $\operatorname{ker} D_{\partial X}=0,\left\{\varphi_{\lambda}, J \varphi_{\lambda} ; \lambda \in \operatorname{spec} D_{\partial X}, \lambda>0\right\}$ forms an orthonormal basis. The lemma follows immediately. 
Now, for the gluing construction, let $\rho(a, b)$ be an increasing $C^{\infty}$ function on $R$ such that $\rho=0$ for $u \leq a$ and $\rho=1$ for $u \geq b$. Define

$$
\begin{array}{rlrl}
\phi_{1} & =\rho\left(\frac{1}{6}, \frac{2}{6}\right), & \psi_{1} & =\rho\left(\frac{3}{6}, \frac{4}{6}\right), \\
\phi_{2} & =1-\rho\left(\frac{5}{6}, 1\right), & \psi_{2}=1-\psi_{1} .
\end{array}
$$

These extend to smooth functions on $X$ in an obvious way. Let $\tilde{D}$ be the Dirac operator on the double of $X$. Then

Lemma 3.4. A parametrix for the heat operator $\partial_{t}+D^{2}(a, T)$ is given by

$$
e=\phi_{1} e^{-t \tilde{D}^{2}} \psi_{1}+\phi_{2}\left(E_{<a}(t)+E_{>a}(t)\right) \psi_{2}
$$

In fact

$$
\left(\partial_{t}+D^{2}(a, T)\right) e=O\left(e^{-C / t}\right) .
$$

Further, one has

$$
e^{-t D^{2}(a, T)}=e+\sum_{m=1}^{\infty}(-1)^{m} c_{m} * e,
$$

where $*$ denotes the convolution of kernels, $c_{1}=\left(\partial_{t}+D^{2}(a, T)\right)$ e, and $c_{m}=c_{m-1} *$ $c_{1}, m \geq 2$.

Proof. This is basically given by the explicit computation, using the standard heat kernel estimate on closed manifolds and the estimate (3.4). Note that (3.8) is the Duhamel principle.

From here one obtains

Proposition 3.5. For all $t>0, e^{-t D^{2}(a, T)}$ is trace class and

$$
\operatorname{Tr}\left(e^{-t D^{2}(a, T)}\right) \sim a_{0} t^{-\frac{n}{2}}+a_{1} t^{-\frac{n-1}{2}}+\cdots+a_{n}+b_{n} \log t+a_{n+1} t^{\frac{1}{2}}+\cdots,
$$

as $t$ goes down to 0 .

Proof. The lemma above together with standard estimates shows that $e^{-t D^{2}(a, T)}$ is a smoothing operator with smooth kernel, hence trace class. Furthermore, (3.8) and 3.4 show

$$
\begin{aligned}
\operatorname{Tr}\left(e^{-t D^{2}(a, T)}\right) & =\int_{X} \operatorname{tr}(e)+O\left(e^{-C / t}\right) \\
& =\int_{X} \phi_{1} \operatorname{tr}\left(e^{-t \tilde{D}^{2}}\right) \psi_{1}+\int_{X} \phi_{2} \operatorname{tr}\left(E_{<a}(t)+E_{>a}(t)\right) \psi_{2}+O\left(e^{-C / t}\right)
\end{aligned}
$$

The first term contributes the standard heat asymptotic on closed manifolds:

$$
\int_{X} \phi_{1} \operatorname{tr}\left(e^{-t \tilde{D}^{2}}\right) \psi_{1} \sim \sum_{i=0}^{\infty} a_{\mathrm{int}, i} t^{-\frac{n-i}{2}},
$$

where $a_{\text {int }, i}=0$ for $i$ odd.

The second term is the boundary contribution, which we analyze according to its natural splitting:

$$
\operatorname{tr}\left(E_{<a}(t)\right) \sim \sum_{i=0}^{\infty} a_{\mathrm{bd}, i}^{<a}(u) t^{-\frac{1-i}{2}}
$$


with $a_{\mathrm{bd}, i}^{<a}(u)$ uniform in $u$. For $E_{>a}(t)$ we use its explicit formula (3.3) and obtain

$$
\int_{\partial X} \operatorname{tr}\left(E_{>a}(t)\right)=2 \sum_{\lambda>a} \frac{e^{-\lambda^{2} t}}{\sqrt{4 \pi t}}-\sum_{\lambda>a} \lambda e^{2 \lambda u} \operatorname{erfc}\left(\frac{u}{\sqrt{t}}+\lambda \sqrt{t}\right) .
$$

Now,

$$
\begin{aligned}
2 \sum_{\lambda>a} \frac{e^{-\lambda^{2} t}}{\sqrt{4 \pi t}} & =\frac{1}{\sqrt{4 \pi t}}\left(\operatorname{Tr}\left(e^{-t D_{\partial X}^{2}}\right)-\sum_{|\lambda|<a} e^{-\lambda^{2} t}\right) \\
& \sim \sum_{i=0}^{\infty} a_{\mathrm{bd}, i}^{>a, 1} t^{-\frac{n-i}{2}} .
\end{aligned}
$$

And

$$
\begin{aligned}
\int_{0}^{\frac{1}{2}} \sum_{\lambda>a} \lambda e^{2 \lambda u} \operatorname{erfc}\left(\frac{u}{\sqrt{t}}+\lambda \sqrt{t}\right) d u \\
\quad=\sum_{\lambda>a}\left\{\frac{1}{2}\left[\operatorname{erfc}\left(\frac{1}{2 \sqrt{t}}+\lambda \sqrt{t}\right) e^{\lambda}-\operatorname{erfc}(\lambda \sqrt{t})\right]-\frac{1}{\sqrt{\pi}} \int_{0}^{\frac{1}{2}} e^{-\lambda^{2} t} e^{-u^{2} / t} d u\right\} \\
\quad=-\frac{1}{\sqrt{\pi}} \sum_{\lambda>a}\left\{e^{-\lambda^{2} t} \int_{0}^{\frac{1}{2 \sqrt{t}}} e^{-v^{2}} \sqrt{t} d v-\int_{\lambda \sqrt{t}}^{\infty} e^{-\xi^{2}} d \xi\right\}+O\left(e^{-C / t}\right) \\
\quad=-\frac{\sqrt{t}}{2}\left(1-O\left(e^{-C / t}\right)\right) \sum_{\lambda>a} e^{-\lambda^{2} t}-\frac{1}{2 \sqrt{\pi}} \int_{t}^{\infty} \sum_{\lambda>a} \lambda e^{-\lambda^{2} s} s^{-\frac{1}{2}} d s+O\left(e^{-C / t}\right) .
\end{aligned}
$$

Since

$$
\sum_{\lambda>a} \lambda e^{-\lambda^{2} s}=\frac{1}{2 \pi i} \int_{\Gamma} \lambda e^{-\lambda^{2} s}\left(\lambda-D_{\partial X}\right)^{-1} d \lambda
$$

where $\Gamma$ is a positively oriented contour in the conical region $\operatorname{Re} \lambda>2 \operatorname{Im} \lambda$ of the complex $\lambda$-plane enclosing exactly all eigenvalues of $D_{\partial X}$ that are larger than $a$. It follows then that

$$
\sum_{\lambda>a} \lambda e^{-\lambda^{2} s} \sim \sum_{i=0}^{\infty} a_{\mathrm{bd}, i}^{>a, 2} s^{-\frac{n-i}{2}}
$$

Combining (3.10), (3.11), (3.12), (3.14), (3.15), (3.16) and (3.17), we obtain the desired asymptotic expansion.

Remark. The problem of the asymptotic expansion of the heat kernel of the APS boundary conditions has been studied extensively in a more general context by Grubb G], Grubb-Seeley GS, and also Brüning-Lesch [BL].

Thus the spectrum of $D_{X}(a, T)$ consists of all eigenvalues of finite multiplicity satisfying the Weyl's asymptotic law. Consequently the eta function

$$
\eta(s, D(a, T))=\sum_{\lambda_{j} \neq 0} \operatorname{sign} \lambda_{j}\left|\lambda_{j}\right|^{-s}
$$

is well-defined for $\operatorname{Re} s>n$. Further by Mellin transform $(\operatorname{Re} s>n)$,

$$
\eta(s, D(a, T))=\frac{1}{\Gamma\left(\frac{s+1}{2}\right)} \int_{0}^{\infty} t^{(s-1) / 2} \operatorname{Tr}\left(D(a, T) e^{-t D^{2}(a, T)}\right) d t .
$$


The following result of [DF] will be of critical importance to us.

Theorem 3.6. For any real number $a^{2} \notin \operatorname{spec}\left(D_{\partial X}^{2}\right)$ and unitary map $T: K_{\partial X}^{+}(a)$ $\rightarrow K_{\partial X}^{-}(a)$, one has

$$
\operatorname{Tr}\left(D(a, T) e^{-t D^{2}(a, T)}\right)=O(1), \text { as } t \rightarrow 0 .
$$

Therefore the eta function $\eta\left(s, D_{X}(a, T)\right)$ is actually holomorphic in the half plane Res $>-\frac{1}{2}$. Consequently the eta invariant $\eta_{X}(a, T)=\eta\left(0, D_{X}(a, T)\right)$ is welldefined and is given by the formula

$$
\eta_{X}(a, T)=\frac{1}{\sqrt{\pi}} \int_{0}^{\infty} t^{-1 / 2} \operatorname{Tr}\left(D_{X}(a, T) e^{-t D_{X}^{2}(a, T)}\right) d t .
$$

\section{Finite propagation SPeEd}

In this section we take a little digression and show that the Dirac operator with the APS type boundary condition has finite propagation speed. As a result, the method of estimating the heat kernel in [CGT] extends to this case. However, this result is not used here.

We follow the elegant treatment of Chernoff [Cf. Thus, let $M$ be a compact spin manifold with boundary, and of product type near the boundary. Fix $a, T$ as in the last section, and denote $D=D(a, T)$ the Dirac operator with the APS type boundary condition. Consider the following symmetric hyperbolic system

$$
\frac{\partial u}{\partial t}=i D u
$$

The finite propagation speed amounts to the following local energy estimate.

Theorem 4.1. Let $u$ be a smooth solution of [4.1) in $[0, T] \times M$. For any $x_{0} \in M$, denote by $B\left(x_{0}, a\right)$ the geodesic ball of radius a centered at $x_{0}$. Then

$$
\int_{B\left(x_{0}, a\right)}\langle u(T), u(T)\rangle d v o l \leq \int_{B\left(x_{0}, a+T\right)}\langle u(0), u(0)\rangle d v o l .
$$

Proof. Define a vector field $Z$ on $[0, T] \times M$ by the prescription

$$
(Z f)(t, x)=\langle u(t), u(t)\rangle f(t, x)-\langle u(t), i c(d f) u(t)\rangle .
$$

Here, as in the statement of the theorem, we use $\langle\cdot, \cdot\rangle$ to denote the inner product on $M$. Note also that $i c(d f)$ is the symbol of $D$ at $d f$. It follows then that $\operatorname{div} Z=0$. Therefore, applying Green's theorem to the domain $K=\{(t, x) \mid 0 \leq t \leq T, x \in$ $\left.B\left(x_{0}, a+t\right)\right\}$ yields

$$
\begin{aligned}
0= & \int_{\partial K}(Z, \nu) d S=\int_{S\left(x_{0}, a\right)}\langle u(T), u(T)\rangle d v o l \\
& -\int_{S\left(x_{0}, a+T\right)}\langle u(0), u(0)\rangle d v o l+\int_{\Sigma}-(Z, \nu) d S,
\end{aligned}
$$

where $\Sigma=\Sigma_{1}+\Sigma_{2}$ and $\Sigma_{1}=\left\{(t, x) \mid 0 \leq t \leq T, x \in \partial B\left(x_{0}, a+t\right)\right\}, \Sigma_{2}=$ $[0, T] \times \partial M \cap K$.

Now on $\Sigma_{1}$, the unit outer normal $\nu$ is proportional to the gradient of the function $\phi=t+d\left(x, x_{0}\right)$. Therefore $\nu=\left(\nu_{0}, \nu^{\prime}\right)$ with $\nu_{0}=\left|\nu^{\prime}\right|$. Hence

$$
(Z, \nu)=\langle u, u\rangle \nu_{0}-\left\langle u, i c\left(\nu^{\prime}\right) u\right\rangle \geq\langle u, u\rangle\left(\nu_{0}-\left|\nu^{\prime}\right|\right)=0,
$$

which shows that $\int_{\Sigma_{1}}(Z, \nu) \geq 0$. 
On the other hand, on $\Sigma_{2}, \nu$ is given by the normal of $\partial M$. Consequently $(Z, \nu)=-\langle u, i J u\rangle=0$ by the boundary condition.

This finishes the proof of the local energy estimate.

Remark. The finite propagation speed property for Dirac operators with APS boundary condition is also discussed in $[\mathrm{L}]$.

\section{The ADIABATIC LIMIT}

In this section we prove the main result of our paper. The strategy is modelled on the proof of Bismut-Cheeger's theorem. Our starting point is Theorem [3.6] which gives an explicit heat kernel representation for the eta invariant. The control for the large time behavior will come from a uniform lower bound on the smallest eigenvalue. For the finite time contribution we show that it splits into an interior part and a boundary part in the adiabatic limit. Remarkably, the boundary contribution turns out to be zero.

Let $Y \rightarrow X \rightarrow B$ be a fibration as in Section 2, but now the base $B$ has boundary. One then has the associated boundary fibration $Y \rightarrow \partial X \rightarrow \partial B$. The adiabatic metric $g_{x} 2.3$ induces an adiabatic metric on the boundary fibration. Let $D_{x}^{\partial X}$ be the Dirac operator on $\partial X$ with respect to the boundary adiabatic metric.

We now make the assumption that the Dirac family along the fibers is invertible. By a result of Bismut-Cheeger [BC1], the eigenvalues of $D_{x}^{\partial X}$ will be uniformly bounded away from zero (see also the proof of Proposition 5.2). In particular, the (classical) APS boundary condition, which corresponds to $a=0$ and $T=0$, is a self-adjoint elliptic boundary condition for $D_{x}=D_{x}^{X}$ (from now on we suppress the superscript $X)$ and from $\S 3$, its eta invariant $\eta\left(D_{x}\right)$ is well-defined.

Theorem 5.1. Under the hypothesis that the Dirac family $D_{Y}$ is invertible, the limit $\lim _{x \rightarrow 0} \bar{\eta}\left(D_{x}\right)=\lim _{x \rightarrow 0} \frac{1}{2} \eta\left(D_{x}\right)$ exists in $\mathbb{R}$ and

$$
\lim _{x \rightarrow 0} \bar{\eta}\left(D_{x}\right)=\int_{B} \hat{A}\left(\frac{R^{B}}{2 \pi}\right) \wedge \tilde{\eta} .
$$

The proof of the theorem occupies the rest of the section. The first step is to show that the smallest eigenvalue of $D_{x}$ is uniformly bounded away from zero. This gives us the desired control for the large time. However, because of the boundary condition, its proof requires considerable care.

Proposition 5.2. Let $D_{x}=D_{x}(0,0)$ denote the Dirac operator $D_{x}$ with the APS boundary condition. Then, under the assumption that the Dirac family along the fibers $D_{Y}$ is invertible, there exists a positive constant $\lambda_{0}$ such that

$$
\left|\lambda_{x}\right| \geq \lambda_{0}
$$

for any eigenvalue $\lambda_{x}$ of $D_{x}$.

Proof. Denote by $($,$) the L^{2}$-metric with respect to the adiabatic metric $g_{x}$. It suffices to show that there is a positive constant $\lambda_{0}$ such that

$$
\left(D_{x} \phi, D_{x} \phi\right) \geq \lambda_{0}^{2}(\phi, \phi),
$$

for all eigensections $\phi=\phi_{x}$ of $D_{x}: D_{x} \phi_{x}=\lambda_{x} \phi_{x}$. The proof is divided in three parts, corresponding to the interior, the boundary, and the patch up. The treatment near the boundary uses a trick from [DW], who attribute it to Werner Müller. 
We first look at the case when $\phi$, not necessarily an eigensection, vanishes near the boundary. This is essentially the argument of Bismut-Cheeger [BC1, since there is no boundary contribution from integration by parts.

Let $\lambda_{1}$ be the smallest of all eigenvalue of $D_{Y}^{2}$, which is positive by our assumption together with the compactness of the base $B$. In equation (2.4) set $E_{x}=\sum_{\alpha} f_{\alpha} \nabla_{f_{\alpha}}^{u}+-\frac{x}{4} \sum_{\alpha \leq \beta} f_{\alpha} f_{\beta} T\left(f_{\alpha}, f_{\beta}\right)$. Then (2.4) becomes $D_{x}=x E_{x}+D_{Y}$. And so

$$
D_{x}^{2}=x^{2} E_{x}^{2}+x\left[E_{x}, D_{Y}\right]+D_{Y}^{2},
$$

with the crucial property that the operator $\left[E_{x}, D_{Y}\right]$ is a first order differential operator which acts fiberwise. Since $D_{Y}$ is invertible, it follows that there is a constant $C$ such that for any section $s$

$$
\left|\int\left\langle\left[E_{x}, D_{Y}\right] s, s\right\rangle\right| \leq C\left\|D_{Y} s\right\|^{2} .
$$

Also, note that

$$
\int_{X}\left\langle E_{x}^{2} \phi, \phi\right\rangle=\int_{X}\left\langle E_{x} \phi, E_{x} \phi\right\rangle \geq 0
$$

since $\phi$ vanishes near the boundary.

Therefore, combining (5.2), (5.3), and again using the fact that $\phi$ vanishes near the boundary, we find

$$
\left(D_{x} \phi, D_{x} \phi\right)=\left(D_{x}^{2} \phi, \phi\right) \geq\left(\lambda_{1}-x C\right)(\phi, \phi) \geq \frac{1}{2} \lambda_{1}(\phi, \phi),
$$

when we take $x \leq \frac{1}{2 C}$.

Now we assume that $\phi$ vanishes in the interior, $\phi \in \operatorname{dom}\left(D_{x}\right)$ and $D_{x} \phi_{x}=\lambda_{x} \phi_{x}$ near the boundary, say over $[0,1] \times \partial X$. On $\partial X$ we have the boundary fibration $Y \rightarrow \partial X \rightarrow \partial B$ with the adiabatic metric $\left.g_{x}\right|_{\partial X}$. The same argument as above implies that the eigenvalues of the boundary Dirac operator $D_{x}^{\partial X}$ is uniformly bounded away from $0:\left|\mu_{x}\right| \geq \frac{1}{2} \lambda_{1}$, when $x$ is small.

Without loss of generality we assume that

$$
\left|\lambda_{x}\right|<\frac{1}{4} \lambda_{1}
$$

Using separation of variables one found that $\phi$ has the following expansion in terms of the spectral resolution of $D_{x}^{\partial X}$ (we now suppress the $x$-dependence of the eigenvalues, i.e. $\mu=\mu_{x} \in \operatorname{spec} D_{x}^{\partial X}$ and $\left.\lambda=\lambda_{x}\right)$ :

$$
\begin{aligned}
\phi= & \sum_{\mu>0} a_{\mu}\left[\frac{\lambda}{\left(\mu^{2}-\lambda^{2}\right)^{\frac{1}{2}}} \sinh \left(\frac{\left(\mu^{2}-\lambda^{2}\right)^{\frac{1}{2}}}{x} u\right) \phi_{\mu}\right. \\
& +\left(\cosh \left(\frac{\left(\mu^{2}-\lambda^{2}\right)^{\frac{1}{2}}}{x} u\right)+\frac{\mu}{\left(\mu^{2}-\lambda^{2}\right)^{\frac{1}{2}}} \sinh \left(\frac{\left(\mu^{2}-\lambda^{2}\right)^{\frac{1}{2}}}{x} u\right) J \phi_{\mu}\right] .
\end{aligned}
$$

Since $\phi$ is in $L^{2}$, the coefficients $a_{\mu}$ are exponentially decaying in $x$ :

$$
\sum_{\mu>0} a_{\mu}^{2} e^{\frac{\mu}{x}} \leq C\|\phi\|^{2}
$$

for some positive constant $C$. 
Now we extend $\phi$ to a section $\tilde{\phi}$ over $\mathbb{R} \times \partial X$ by defining it to be $\sum_{\mu>0} a_{\mu} e^{\mu u} J \phi_{\mu}$ for $u<0$. This extension lies in the Sobolev space $H^{1}$, with its $L^{2}$-norm

$$
\|\phi\|^{2} \leq\|\tilde{\phi}\|^{2} \leq\|\phi\|^{2}+\frac{1}{2 \mu_{1}} \sum a_{\mu}^{2} \leq\left(1+e^{-\frac{\mu_{1}}{x}}\right)\|\phi\|^{2},
$$

using (5.6). Since the spectrum of $\left(J\left(x \partial_{u}+D_{x}^{\partial X}\right)\right)^{2}=-x^{2} \partial_{u}^{2}+\left(D_{x}^{\partial X}\right)^{2}$ is bounded below by the smallest eigenvalue of $\left(D_{x}^{\partial X}\right)^{2}$, which is in turn uniformly bounded by $\frac{1}{4} \lambda_{1}^{2}$, we apply the min-max principle to obtain

$$
\left(D_{x} \phi, D_{x} \phi\right)=\left(D_{x} \tilde{\phi}, D_{x} \tilde{\phi}\right) \geq \frac{1}{4} \lambda_{1}^{2}(\tilde{\phi}, \tilde{\phi}) \geq \frac{1}{4} \lambda_{1}^{2}(\phi, \phi) .
$$

Finally, we use a patching argument to deal with the general case. Let $n=\left[\frac{1}{\sqrt{x}}\right]$ and $u_{i}=\frac{i}{n}$. Since

$$
\left(\phi_{x}, \phi_{x}\right) \geq \int_{0}^{1}\left\langle\phi_{x}, \phi_{x}\right\rangle \frac{d u}{x} d y=\sum_{0}^{n-1} \int_{u_{i}}^{u_{i+1}}\left\langle\phi_{x}, \phi_{x}\right\rangle \frac{d u}{x} d y
$$

it follows that there exists an $i$ such that

$$
\int_{u_{i}}^{u_{i+1}}\left\langle\phi_{x}, \phi_{x}\right\rangle \frac{d u}{x} d y \leq \frac{1}{n}\left(\phi_{x}, \phi_{x}\right) .
$$

Now let $f(u)$ be a smooth function on $[0,1]$ such that $f=1$ for $u \leq u_{i}$ and $f=0$ for $u \geq u_{i+1}$ and $\left|f^{\prime}\right| \leq 2 n$. Then

$$
\begin{aligned}
\left(D_{x} \phi_{x}, D_{x} \phi_{x}\right)= & \left(D_{x}\left(f \phi_{x}\right), D_{x}\left(f \phi_{x}\right)\right)+\left(D_{x}\left((1-f) \phi_{x}\right), D_{x}\left((1-f) \phi_{x}\right)\right) \\
& +2\left(D_{x}\left(f \phi_{x}\right), D_{x}\left((1-f) \phi_{x}\right)\right) \\
\geq & \lambda_{0}\left(\left(f \phi_{x}, f \phi_{x}\right)+\left((1-f) \phi_{x},(1-f) \phi_{x}\right)\right) \\
& -6 x^{2}\left(\max \left|f^{\prime}\right|\right)^{2}\left(\frac{1}{n}\right)\left(\phi_{x}, \phi_{x}\right)-6\left(D_{x} \phi_{x}, D_{x} \phi_{x}\right) .
\end{aligned}
$$

The choice of $i$ and $f$ is such that

$$
\left(f \phi_{x}, f \phi_{x}\right)+\left((1-f) \phi_{x},(1-f) \phi_{x}\right) \geq\left(1-\frac{2}{n}\right)\left(\phi_{x}, \phi_{x}\right) .
$$

Therefore, we have by combining,

$$
7\left(D_{x} \phi_{x}, D_{x} \phi_{x}\right) \geq\left(\lambda_{0}-5 x\right)\left(\phi_{x}, \phi_{x}\right)
$$

As a consequence of this uniform estimate of the eigenvalue and the formula (3.21) we have, for any $T>0$,

$$
\eta\left(D_{x}\right)=\frac{1}{\sqrt{\pi}} \int_{0}^{T} t^{-1 / 2} \operatorname{Tr}\left(D_{x} e^{-t D_{x}^{2}}\right) d t+O\left(e^{-\lambda_{0}^{2} T}\right) .
$$

This reduces the study to the finite time behavior of the heat kernel.

Now recall that a parametrix of the heat operator is obtained in (3.6) by the patching construction. In the adiabatic limit we have

Lemma 5.3. There exists a constant $C^{\prime}$ such that

$$
e^{-t D^{2}(a, T)}=\phi_{1} e^{-t \tilde{D}^{2}} \psi_{1}+\phi_{2}\left(E_{<a}(t)+E_{>a}(t)\right) \psi_{2}+O\left(x^{n} e^{-C^{\prime} / x^{2} t}\right)
$$


and

$$
D(a, T) e^{-t D^{2}(a, T)}=\phi_{1} \tilde{D} e^{-t \tilde{D}^{2}} \psi_{1}+\phi_{2} D\left(E_{<a}(t)+E_{>a}(t)\right) \psi_{2}+O\left(x^{n} e^{-C^{\prime} / x^{2} t}\right) .
$$

Proof. This follows from Lemma 3.4 and Lemma 3.3. In fact (using the notation in (3.8)

$$
c_{1}=\phi_{1}^{\prime} J e^{-t \tilde{D}^{2}} \psi_{1}+\phi_{2}^{\prime} J\left(E_{<a}(t)+E_{>a}(t)\right) \psi_{2} .
$$

Since the support of $\phi_{i}^{\prime}(i=1,2)$ is away from that of $\psi_{i}$ (by a definite distance), therefore, with respect to the adiabatic metric, we have from Lemma 3.3 that

$$
\left|c_{1}\right| \leq C t^{-\frac{n}{2}} e^{-\frac{1}{144 x^{2} t}}
$$

Hence

$$
e^{-t D^{2}(a, T)}=e+O\left(t^{-\frac{n}{2}} e^{-\frac{1}{144 x^{2} t}}\right)=e+O\left(x^{n} e^{-\frac{1}{288 x^{2} t}}\right) .
$$

The second formula is proved similarly.

Now we can finish the proof of Theorem [5.1. By formulae (5.9), (5.8), one deduces

$$
\begin{aligned}
\eta\left(D_{x}\right)= & \frac{1}{\sqrt{\pi}} \int_{0}^{T} t^{-1 / 2} \operatorname{Tr}\left(\phi_{1} \tilde{D}_{x} e^{-t \tilde{D}_{x}^{2}} \psi_{1}\right) d t \\
& +\frac{1}{\sqrt{\pi}} \int_{0}^{T} t^{-1 / 2} \operatorname{Tr}\left(\phi_{2} D_{x} E_{x}(t) \psi_{2}\right) d t+O\left(e^{-\lambda_{0}^{2} T}\right),
\end{aligned}
$$

where $\tilde{D}_{x}$ is the Dirac operator on the double of $X$ and $E_{x}(t)$ is the heat kernel on the infinite cylinder. Because of (3.5) the second term in (5.11) drops out. On the other hand, from the work of [BC1], we have

$$
t^{-\frac{1}{2}} \operatorname{tr}\left(\tilde{D}_{x} e^{-\tilde{D}_{x}^{2} t}\right)=\frac{\sqrt{\pi}}{(2 \pi i)^{k}} \int_{B} \hat{A}\left(i R^{B}\right) \operatorname{tr}^{\text {even }}\left(\left(D_{Y}+\frac{c(T)}{4 t}\right) e^{-B_{t}^{2}}\right)+O\left(x\left(1+T^{N}\right)\right)
$$

for some $N$, uniformly in $x$. Here we state the result for the case when the fiber has odd dimension. In the case when the fiber has even dimension one has the same result except that $\operatorname{tr}^{\text {even }}\left(\left(D_{Y}+\frac{c(T)}{4 t}\right) e^{-B_{t}^{2}}\right)$ will be replaced by $\operatorname{tr}_{s}\left(\left(D_{Y}+\frac{c(T)}{4 t}\right) e^{-B_{t}^{2}}\right)$.

By the product nature of the metric in the collar neighborhood of $\partial B$ and $\partial X$, neither $\hat{A}\left(i R^{B}\right)$ nor $\operatorname{tr}^{\text {even }}\left(\left(D_{Y}+\frac{c(T)}{4 t}\right) e^{-B_{t}^{2}}\right)$ will produce a term involving $d u$. Thus we can safely throw away the cut-off functions in (5.11) and obtain

$$
\begin{aligned}
\eta\left(D_{x}\right)= & \frac{1}{(2 \pi i)^{k}} \int_{B} \hat{A}\left(i R^{B}\right) \int_{0}^{T} t^{-1 / 2} \operatorname{Tr}^{\text {even }}\left(\left(D_{Y}+\frac{c(T)}{4 t}\right) e^{-B_{t}^{2}}\right) d t \\
& +O\left(x\left(1+T^{N}\right)\right)+O\left(e^{-\lambda_{0}^{2} T}\right)+O\left(x^{n} e^{-C^{\prime} / x^{2} t}\right) .
\end{aligned}
$$

Taking $T=|\log x|$ and letting $x \rightarrow 0$, we obtain the desired formula.

\section{REFERENCES}

[ADS] M. F. Atiyah, H. Donnelly, I. M., Singer, Eta invariants, signature defect of cusps and values of L-functions. Ann. of Math. 118 (1983), 131-177. MR 86g:58134a

[APS] M. F. Atiyah, V. Patodi, I. M. Singer, Spectral asymmetry and Riemannian geometry, I, Math. Proc. Cambridge Philos. Soc. 77 (1975), 43-69. MR 48:9776

[BGV] N. Berline, E. Getzler, M. Vergne, Heat kernels and Dirac operators, Springer-Verlag, Berlin, 1992. MR 94e:58130

[B] Bismut, J.-M.: The Atiyah-Singer index theorem for families of Dirac operators: two heat equation proofs. Invent. Math. 83 (1986), 91-151. MR 87g:58117 
[BF1] J.-M. Bismut, D. Freed, The analysis of elliptic families, I. Comm. Math. Phys. 106 (1986), 159-176. MR 88h:58110a

[BF2] J.-M. Bismut, D. Freed, The analysis of elliptic families, II, Comm. Math. Phys. 107 (1986), 103-163. MR 88h:58110b

[BC1] J.-M. Bismut, J. Cheeger, $\eta$-invariants and their adiabatic limits, Journal of A. M. S. 2 (1989), 33-70. MR 89k:58269

[BC2] J.-M. Bismut, J. Cheeger, Families index theorem for manifolds with boundary, I, J. Functional Analysis, 89 (1990), 313-363. MR 91e:58180

[BC3] J.-M. Bismut, J. Cheeger, Families index theorem for manifolds with boundary, II, J. Functional Analysis, 90 (1990), 306-354. MR 91e:58181

[BC4] J.-M. Bismut, J. Cheeger, Trangressed Euler classes of $S L(2 n, \mathbb{Z})$ vector bundles, adiabatic limits of eta invariants and special values of L-functions, Ann. Scient. Éc. Norm. Sup. 25 (1992), 335-391. MR 94e:57042

[BL] J. Brüning, M. Lesch, On the eta invariant of certain nonlocal boundary problems, Duke Math. J., 96(1999), 425-468. MR 99m:58180

[BW] B. Booss and K. Wojciechowski, Elliptic boundary problems for Dirac operators, Birkhäuser, 1993.

[C1] J. Cheeger, $\eta$-invariants, the adiabatic approximation and conical singularities, J. Diff. Geom. 26 (1987), 175-221. MR 89c:58123

[C2] J. Cheeger, Spectral geometry of singular Riemannian spaces, J. Diff. Geom., 18(1983), 575-657. MR 85d:58083

[CGT] J. Cheeger, M. Gromov, M. Taylor, Finite propagation speed, kernel estimates for functions of the Laplace operator, and the geometry of complete Riemannian manifolds, J. Diff. Geom., 17(1982), 15-53. MR 84b:58109

[Cf] P. Chernoff, Essential self-adjointness of powers of generators of hyperbolic equations, J. Funct. Anal., 12(1973), 401-414. MR 51:6119

[D] X. Dai, Adiabatic limits, the non-multiplicativity of signature and Leray spectralsequence, Journal of A. M. S. 4 (1991) 265-321. MR 92f:58169

[DF] X. Dai, D. Freed, Eta invariants and determinant lines, J. Math. Phys., 35(1994), 51555194. MR 96a:58204

[DZ] X. Dai, W. Zhang, Circle bundles and the Kreck-Stolz invariant, Trans. A. M. S., 347(1995), pp 3587-3593. MR 96d:58132

[DW] R. D. Douglas, K. P. Wojciechowski Adiabatic limits of the $\eta$-invariants: the odd dimensional Atiyah-Patodi-Singer problem, Commun. Math. Phys. 142 (1991) 139-168. MR 92j:58110

[G] G. Grubb, Heat operator trace expansions and index for general Atiyah-Patodi-Singer boundary problems, Comm. P. D. E., 17(1992), 2031-2077. MR 94a:58186

[GS] G. Grubb, R. Seeley, Weakly parametric pseudodifferential operators and Atiyah-PatodiSinger boundary problems, Invent. Math., 121(1995), 481-529. MR 96k:58216

[KW] Klimek, K. Wojciechowski, $\eta$-invariants on manifolds with cylindrical ends, Diff. Geom. and its Appl., 3(1993), 191-201. MR 94k:58163

[LW] M. Lesch, K. P. Wojciechowski, On the $\eta$-invariant of generalized Atiyah-Patodi-Singer boundary value problems, Illinois J. Math., 40(1996), 30-46. MR 97d:58194

[L] M. Lesch, Operators of Fuchs Type, Conical Singularities, Asymptotic Methods, TeubnerTexte zur Mathematik, 136, Stuttgart, 1997. MR 98d:58174

[M] R. Melrose, The Atiyah-Patodi-Singer Index Theorem, A. K. Peters, 1993. MR 96g:58180

$[\mathrm{MM}]$ R. Mazzeo, R. Melrose, The adiabatic limit, Hodge cohomology and Leray's spectral sequence of a fibration, J. Diff. Geom., 31(1990), 185-213. MR 90m:58004

[MP1] R. Melrose, P. Piazza, Families of Dirac operators, boundaries and the b-calculus, Jour. Diff. Geom. 46(1997), 99-180. MR 99a:58144

[MP2] R. Melrose, P. Piazza, An index theorem for families of Dirac operators on odd-dimensional manifolds with boundary, Jour. Diff. Geom. 46(1997), 287-334. MR 99a:58145]

[Mu1] W. Müller, Signature defects of cusps of Hilbert moldular varieties and values of L-series at $s=1$, J. Diff. Geom. 20 (1984), 55-119. MR 87c:11048

[Mu2] W. Müller, Eta invariants and manifolds with boundary, J. Diff. Geom., 40(1994), 311-377. MR 96c:58165

[N] L. Nicolaescu, Adiabatic limits of the Seiberg-Witten equations on Seifert manifolds, preprint. 
[Se] R. T. Seeley, Topics in pseudo-differential operators, CIME, Edizioni Cremonese, Roma 1969, 169-305. MR 41:3973

[Si] I. M. Singer, The eta invariant and the index, Mathematical aspects of string theory (S. T. Yau ed.) World Scientific, Singapore, 1987. MR 89m:81001

[W] E. Witten, Global gravitational anomalies, Comm. Math. Phys. 100 (1985) 197-229. MR 87k:58282

[Z] Zhang, W., Circle bundles, adiabatic limits of $\eta$-invariants and Rokhlin congruences, Ann. Inst. Fourier, Grenoble, 44 (1994), 249-270. MR 95h:58127

Department of Mathematics, University of California, Santa Barbara, California 93106

E-mail address: dai@math.ucsb.edu 